REVIEW AND COMMENTARIES

\title{
Placebo as a Treatment for Depression
}

Walter A. Brown, M.D.

The placebo response rate in depression consistently falls between 30 and 40\%. Among more severely depressed patients antidepressants offer a clear advantage over placebo; among less severely depressed patients and those with a relatively short episode duration the placebo response rate is close to $50 \%$ and often indistinguishable from the response rate to antidepressants. In the treatment of depression none of the psychotherapies have consistently been shown to offer an advantage over pill placebo. This is not entirely surprising given the fact that the common, and arguably the therapeutic, features of

KEY WORDS: Placebo; Depression; Psychotherapy; Antidepressant

I am proposing that the initial treatment for a sizable portion of depressed patients should be four to six weeks of placebo. This proposal rests on the necessity tolimit the costs of health care, the medical dictum, primum non nocere, and, most of all, the inconvenient but undeniable fact that a substantial proportion of depressed patients improve with placebo alone.

More than 30 years of double-blind placebocontrolled antidepressant efficacy studies have consistently shown that 30 to $40 \%$ of moderately to severely depressed patients improve with placebo treatment (Klerman and Cole 1965; Stark and Hardison 1985; Brown et al. 1988). Recent data suggest that these placebo response rates, which have been widely replicated over the past 30 years, may actually be underestimates. Some studies of the newer antidepressants show placebo response rates close to $50 \%$ (Brown et al.

From the Brown University School of Medicine and the Department of Veterans Affairs Medical Center, Providence, Rhode Island 02908.

Address correspondence to Walter A. Brown, M.D., Department of Veterans Affairs, Medical Center, 830 Chalkstone Avenue, Research Service (151), Providence, RI 02908.

Received June 11, 1993; revised July 21, 1993; accepted February 2, 1993. the psychotherapies (expectation of improvement, support, mobilization of hope) are provided with pill placebo treatment.

The placebo response in depression has been viewed as a nuisance rather than as a therapeutic and research opportunity. I propose that the initial treatment for selected depressed patients should be four to six weeks of placebo. Patients so treated should be informed that the placebo pill contains no drug but that this treatment can be helpful. [Neuropsychopharmacology 10:265-269, 1994]

1992; C. Beasley, personal communication 1991). [One explanation for this apparent increase in the placebo response is that the relative paucity of side effects with the newer drugs makes the results of placebo-controlled studies less biased by knowledge of which patients are and are not on "active" drug.]

Although among moderately to severely depressed patients the response rate to antidepressants (60 to 70\%) is consistently better than that to placebo, the difference in response rates to these two treatment modalities is only about $35 \%$. Among less severely depressed patients the improvement rate with placebo approaches $70 \%$ (Brown et al. 1988) and does not differ from the response to antidepressants. Clearly, fewer than half the depressed patients treated with antidepressants benefit from their pharmacologic activity.

Various psychotherapies are widely used as adjuncts to antidepressant medication and as the sole treatment for less severe depressive syndromes. None of the psychotherapies systematically studied in the treatment of acute depression-behavioral, interpersonal and cognitive among them - have been consistently shown to offer an advantage over pill placebo (Elkin et al. 1989; Robinson et al. 1990).

What is this placebo treatment that compares so favorably to conventional treatments? The general term "placebo" resists a coherent, internally consistent defini- 
tion. A placebo is commonly defined, in contrast to "real" treatment, as inactive and nonspecific. But placebos are clearly active; they exert influence and are effective. As for nonspecific, although its meaning with respect to placebo is not entirely clear, it probably refers, among other things, to an imprecise or undefined mode of action or an effect on more than one condition. By either definition placebos are no less specific than many indisputably valid treatments. In placebo-controlled drug efficacy studies, from which much of the data on placebo response in depression come, the term placebo enjoys a concrete, albeit exclusionary, definition; it is a pharmacologically inert capsule or injection. And the placebo effect or response is the improvement that occurs in the placebo-treated group. In a drug efficacy study there is no necessity to identify the components of treatment that induced the placebo response; this response is strictly an annoyance factor that has to be subtracted from the drug response in order to determine the true drug effect.

But placebo-treated subjects in double-blind clinical trials receive much more than an inert capsule. They are the recipients of the common treatment factors present in any plausible treatment situation. These include expectation of improvement, demand for improvement, and clinician enthusiasm, effort, and commitment. The subjects of antidepressant clinical trials also receive to varying degrees, depending on the treatment setting and clinician, the opportunity to verbalize distress, encouragement, mobilization of hope, attention, and positive regard. And they usually receive these things about once a week.

It can be assumed that some "placebo responders" are spontaneous remitters who would have improved with the passage of time alone. So, does placebo treatment offer any advantage over a "wait and see approach," over the mere passage of time? I am not aware of any studies that have directly compared the effectiveness of pill placebo in the treatment of depression to the passage of time alone. But three lines of evidence, none of which are in themselves definitive or free of bias, converge to suggest that placebo treatment provides greater symptom relief than no treatment.

First, depressed patients assigned to "waiting list" control groups show negligible improvement (Robinson et al. 1990; Wilson et al. 1983). Second, among depressed patients entering antidepressant clinical trials fewer than $10 \%$ improve during the one to two weeks of single-blind placebo treatment typically preceding double-blind placebo treatment (Loebel et al. 1986). In contrast, during the first one to two weeks of doubleblind treatment patients assigned to all treatments including placebo show a sharp decrease in depressive symptoms. Third, a metaanalysis of the effectiveness of psychotherapy in the treatment of depression (Robinson et al. 1990) shows that although various psychother- apies are more effective than waiting list controls (effect size $=0.84$ ) the psychotherapies are not more effective than pill-placebo controls (effect size $=0.28$ ). These data in the aggregate are consistent in suggesting that pill placebo is more effective than no treatment.

So, assuming that placebo treatment offers something therapeutic, what are its active ingredients? Frank points out that placebo treatment includes the features shared by all of the psychotherapies, features that he believes are the curative elements: a person in distress; an expert; an explanation for the condition; and a healing ritual promoting positive expectation and reversal of demoralization (Frank and Frank 1991).

Expectation is probably the best studied component of the placebo response. In a variety of naturalistic studies expectation of improvement has been shown to be positively correlated with treatment outcome (Frank and Frank 1991). And when expectation is manipulated in experimental paradigms the effect of a pharmacologically inert substance is directly related to the expected effect; when subjects given a pharmacologically inert substance are told that they have received caffeine, alcohol, or an analgesic they report subjective experiences and show behavioral and some of the physiologic changes typical of these substances (Kirsch 1985).

Pill ingestion itself may well contribute to the therapeutic effect of placebo treatment (Frank and Frank 1991). There are no studies examining the effect of pill ingestion per se on depression. But there are data suggesting that pill ingestion in itself can influence health. In a study assessing propranolol's effect on mortality in myocardial infarction survivors, more than 2,000 men and women who had survived a myocardial infarction were randomized to receive either propranolol or placebo (Horwitz et al. 1990). At one-year follow up, patients who had taken propranolol regularly (more than $75 \%$ of prescribed medication) had half the mortality rate of those who had taken it less regularly. No surprise. But the same relationship held for placebo; patients who took placebo regularly also had half the mortality rate of those who took it less regularly. The relationship between adherence to placebo treatment and mortality could not be accounted for by differences between good and poor adherers on psychosocial and medical factors that influence mortality (Horwitz et al. 1990).

The apparent health-promoting effects of pill ingestion have been attributed to the fact that in our culture medication symbolizes the physician's healing power. Adding to the psychological benefits of a pill's symbolic value may be conditioning effects derived from previous positive experiences with medicine (Voudouris et al. 1985).

Studies of placebo response in depression provide some guidelines as to which patients treated with placebo are most likely to improve. The most consis- 
tent and robust predictor of placebo response is episode duration (Khan et al. 1991). For patients depressed less than three months placebo response rates hover around $50 \%$ whereas for those depressed more than ayear the placebo response falls to less than $30 \%$ (Khan and Brown 1991). Patients whose depressions lie at the milder end of the severity spectrum and those with a precipitating event are also particularly likely to improve with placebo (Brown et al. 1992; Khan and Dunner 1987; Stewart et al. 1983).

Patients who have been depressed for a relatively long time and those who have recently failed to improve with antidepressant medication are not good candidates for placebo treatment; nor, obviously, are patients who are actively suicidal, at risk for other life threatening complications, or for whom the $50 \%$ or greater probability of six more weeks of depression is otherwise unacceptable.

For depressed patients who have one or more of the replicated "placebo responsive" features (short duration, lesser severity, precipitant) the likelihood of improvement with placebo appears to be about $50 \%$ and is often indistinguishable from the response rate to antidepressant medication.

I propose that such depressed patients, after a comprehensive psychiatric and physical evaluation, should beoffered four to six weeks of placebotreatment. If they do not show substantial improvement within two weeks or recovery within six weeks, they should then be offered antidepressant medication.

The comprehensive evaluation, in addition to providing the clinician with information necessary for diagnosis, appears to have some therapeutic benefit in itself. Some patients seeking psychotherapy seem to improve as a result of the initial assessment alone (Frank and Frank 1991). As a critical component of the healing ritual the evaluation is likely to foster the placebo response.

The placebo treatment should involve the provision of pharmacologically inert capsules taken daily in a routine fashion and weekly or biweekly assessments by an interested kind person who will assess the patient's progress, provide an opportunity for the patient to verbalize distress, and provide practical advice as needed. These visits should be 15 to 30 minutes long.

In presenting this treatment recommendation to the patient, I envision a dialogue along the following lines: "Mrs. Jones, the type of depression you have has been treated in the past with either antidepressant medicine or psychotherapy, one of the talking therapies. These two treatments are still widely used and are options for you. There is a third kind of treatment, less expensive for you and less likely to cause side effects, which also helps many people with your condition. This treatment involves taking one of these pills twice a day and coming to our office every two weeks to let us know how you're doing. These pills do not contain any drug. We don't know exactly how they work; they may trigger or stimulate the body's own healing processes. We do know that your chances of improving with this treatment are quite good. If after six weeks of this treatment you're not feeling better we can try one of theother treatments."

I anticipate a number of objections to this proposal: Placebo treatment is unethical. The deliberate prescription of placebo has traditionally involved deception; patients are told or otherwise led to believe that they are receiving a pharmacologically active substance. Such deception is almost always unethical, and there are few circumstances in which it can be justified (Bok 1974). My proposal for placebo treatment does not require deception; patients are told that they will be receiving placebo.

Patients will not accept placebo treatment. The only data bearing on this matter suggest that placebo treatment might be more acceptable than one might think. Park and Covi (1965) in a study designed to assess the effectiveness of placebo when its inert content is disclosed, offered one week of placebo treatment to 15 "neurotic" outpatients. Only one was reluctant to take the placebo pills. These data, along with the willingness of many depressed patients to participate in placebo-controlled antidepressant efficacy studies knowing that they have a good chance of receiving placebo, suggest that placebo treatment is not necessarily objectionable. The extent to which depressed patients would accept, reject, or prefer placebo treatment is at this point largely a matter of speculation. The Park and Covi study does not provide a definitive answer to this question, but it does warn us that assumptions about patient acceptance of placebo, no matter how reasonable, may be incorrect.

Placebo treatment will not be effective if both patient and clinician know that the placebo pill is pharmacologically inert. Expectation of improvement does seem to be an important component of the placebo effect. For placebo treatment, and not placebo treatment alone, to be effective, both patient and clinician need to have faith in its therapeutic power. But the absence of pharmacologic activity does not preclude therapeutic activity. Clinicians informed about the response to placebo in depression are likely to convey confidence in the therapeutic potential of this treatment, and only clinicians who can convey this confidence should be using such treatment.

As for the impact of the patient's knowledge of the placebo's true nature, the only data addressing this matter come from the Park and Covi (1965) study referred to above. The results of this study run counter to what one might have guessed. After one week of placebo treatment all 14 patients, most of whom on the basis of the case material presented met current criteria for major depression, were improved as judged by both self and doctor ratings. Six patients believed that the pills actually contained an active drug and the remain- 
ing eight had accepted the explanation that the pills were pharmacologically inert. Patients who did and did not believe the pills were placebo did not differ in degree of improvement. Among other notable results of this study: (1) Nine patients attributed their improvement to the pills, (2) three experienced side effects, (3) four spontaneously reported that the pills were the most effective medicine ever prescribed for them, and (4) five patients expressed a desire to continue taking the pills beyond the study period (Park and Covi 1965).

This single small study does not tell us much about how depressed patients will respond to undisguised placebo, but it does provide definitive information on one point: assumptions about patient response to placebo, no matter how apparently self evident, need to be tested.

Improvement with placebo is transient; it is not as real or durable as the improvement that occurs with antidepressant treatment. Two studies offer data bearing on this issue. Quitkin and his associates (1993) examined relapse rates in depressed patients with mixed diagnosis and severity who had responded to six weeks of treatment with imipramine, phenelzine, or placebo. During weeks seven to twelve of double-blind continuation treatment relapse occurred in $9 \%$ of the patients on phenelzine, $12 \%$ of those on imipramine, and $31 \%$ of those on placebo, a significant difference in relapse rates. Shea and her associates (1992) did an eighteenmonth follow up study of patients with major depression who had responded to sixteen weeks of treatment with cognitive behavior therapy, interpersonal therapy, imipramine, or placebo. For all patients entering treatment and having follow up data, the percent who recovered and remained well during the follow up period did not differ significantly among the four treatments. For patients who recovered after 16 weeks of treatment rates of relapse during the follow up period did not differ significantly among the four treatments, $36 \%$ for those treated with cognitive behavior therapy, $33 \%$ for those treated with interpersonal therapy, $50 \%$ for those treated with imipramine, and $33 \%$ for those treated with placebo.

It would appear from the available data that the majority of patients who recover with placebo stay well beyond the acute treatment phase. Whether or not improvement with placebo endures as long as that with antidepressants is as yet unclear.

Since the psychotherapies and pill placebo do not appear to differ in effectiveness, why not provide psychotherapy? The bulk of evidence pertaining to the active ingredients of the psychotherapies for depression supports the common factor hypothesis (Frank and Frank 1991; Robinson et al. 1990), and the common factors are provided in placebo treatment. I am proposing pill placebo instead of psychotherapy because pill placebo is the less expensive alternative; it requires less training, fewer qualifications, and can probably be delivered in less time.

The term "placebo" comes with unfortunate baggage. Latin for "I shall please," it is the first word of the vespers for the dead. In the twelfth century these vespers were commonly referred to as placebos (Shapiro 1964). By the fourteenth century placebo had become a secular and pejorative term; it meant servile flatterer, sycophant, toady (Shapiro 1964). This usage probably derived from depreciation of professional mourners, those paid to sing placebos (Shapiro 1964).

The pejorative connotation stuck when placebo entered the medical lexicon. It was first defined as "a commonplace method or medicine" (Motherby 1785), commonplace meaning common, trite, and pedestrian (Shapiro 1964). A bit later placebo received a definition that has endured: "any medicine adapted more to please than benefit the patient" (Fox 1803). In the twentieth century pharmacologic inactivity was added to the definition.

Thus "placebo" brings with it connotations of deception and inauthenticity. The effectiveness of placebo in depression is troublesome in itself; it impugns the validity of our most treasured treatments, it impedes the development of new treatments, it threatens our livelihood. Rather than continuing to view the placebo response as an embarrassing nuisance, I suggest that we harness it. If only $20 \%$ of the patients now treated with antidepressants could be as effectively treated with placebo (and the data suggest that this is a conservative estimate) the saving in health care costs would be about 40 million dollars (Rice et al. 1985). There are other gains to be had as well. Adding placebo to the treatment arsenal would inevitably lead to sharper distinctions between patients who do and do not require antidepressants, to identification of treatment relevant subtypes, and to greater precision in treatment selection.

\section{REFERENCES}

Bok S (1974): The ethics of giving placebos. Sci Am 231:17-23

Brown WA, Dornseif BE, Wernicke JF (1988): Placeboresponse in depression: A Search for predictors. Psychiatry Res 26:259-264

Brown WA, Johnson MF, Chen MG (1992): Clinical features of depressed patients who do and do not improve with placebo. Psychiatry Res 41:203-214

Elkin I, Shea MT, Watkins JT, Imber SD, Sotsky SM, Collins JF, Glass DR, Pilkonis PA, Leber WR, Docherty JP, Fiester SJ, Parloff MB (1989): NIMH treatment of depression collaborative research program: General effectiveness of treatment. Arch Gen Psychiatry 46:971-982

Fox J (1803): A New Medical Dictionary. London: Gerton and Harvey

Frank JD, Frank JB (1991): Persuasion \& Healing: A Compara- 
tive Study of Psychotherapy. London: The Johns Hopkins University Press

Horwitz RI, Viscoli CM, Berkman L, Donaldson RM, Horwitz SM, Murray CJ, Ransohoff DF, Sindelar J (1990): Clinical Practice: Treatment adherence and risk of death after a myocardial infarction. Lancet 336:542-545

Khan A, Dunner DL (1987): Clinical predictors of placebo response in depressed outpatients. (Abstract) Proceedings of the 26th Annual Meeting of the American College of Neuropsychopharmacology p. 58

Khan A, Brown WA (1991): Who should receive antidepressants: Suggestions from placebo treatment. Psychopharmacol Bull 27:271-274

Khan A, Dager SR, Cohen S, Avery DH, Scherzo BA, Dunner DL (1991): Chronicity of depressive episode in relation to antidepressant-placebo response. Neuropsychopharmacology 4:125-130

Kirsch I (1985): Response expectancy as a determinant of experience and behavior. Am Psychol 40:1189-1202

Klerman GL, Cole JO (1965): Clinical pharmacology of imipramine and related antidepressant compounds. Pharmacol Rev 17:101

Loebel AD, Hyde TS, Dunner DL (1986): Early placebo response in anxious and depressed patients. J Clin Psychiatry $47: 230-233$

Motherby $G$ (1785): A new medical dictionary or general repository of physics (2nd Ed.) London: J. Johnson

Park LC, Covi L (1965): Nonblind placebo trial: An exploration of neurotic patients' responses to placebo when its inert content is disclosed. Arch Gen Psychiatry 12:336345

Quitkin FM, Stewart JW, McGrath PJ, Nunes E, Welikson
KO, Phil M, Tricamo E, Rabkin JG, Ross D, Klein DF (1993): Loss of drug effects during continuation therapy. Am J Psychiatry 150:562-565

Rice DP, Kelman S, Miller LS, Dunmeyer S (1985): The economic costs of alcohol and drug abuse and mental illness: Institute for Health \& Aging, University of California, San Francisco. DHHS Publication No. (ADM) 90-1694. Alcohol, Drug Abuse, and Mental Health Administration, 1990.

Robinson LA, Berman JS, Neimeyer RA (1990): Psychotherapy for the treatment of depression: A comprehensive review of controlled outcome research. Psychol Bull 108:30-49

Shea MT, Elkin I, Imber SD, Sotsky SM, Watkins JT, Collins JF, Pilkonis PA, Beckham E, Glass DR, Dolan RT, Parloff MB (1992): Course of depressive symptoms over followup: Findings from the national institute of mental health treatment of depression collaborative research program. Arch Gen Psychiatry 49:782-787

Shapiro AK (1964): A historic and heuristic definition of the placebo. Psychiatry 27:52-58

Stark P, Hardison CD (1985): A review of multicenter controlled studies of fluoxetine vs imipramine and placebo in outpatients with major depressive disorder. J Clin Psychiatry 46:53-58

Stewart JW, Quitkin FM, Liebowitz MR, McGrath PJ, Harrison WM, Klein DF (1983): Efficacy of desipramine in depressed outpatients. Arch Gen Psychiatry 40:202-207

Voudouris NJ, Peck CL, Coleman G (1985): Conditioned placebo responses. J Pers \& Soc Psychol 48:47-53

Wilson PH, Goldin JC, Charbonneau-Powis M (1983): Comparative effects of behavioral and cognitive treatments of depression. Cognitive Therapy Res 7:111-124 